“C 2017 IEEE. Personal use of this material is permitted. Permission from IEEE must be obtained for all other uses, in any current or future media, including reprinting/republishing this material for advertising or promotional purposes, creating new collective works, for resale or redistribution to servers or lists, or reuse of any copyrighted component of this work in other works." 


\title{
Diversity Combining for Layered Asymmetrically Clipped Optical OFDM Using Soft Successive Interference Cancellation
}

\author{
Thomas Q. Wang, Hang Li, and Xiaojing Huang, Senior Member, IEEE
}

\begin{abstract}
In this letter, we present a novel diversity combining receiver for demodulating layered asymmetrically clipped optical orthogonal frequency division multiplexing (ACO-OFDM). The receiver consists of a soft successive interference cancellation (SIC) module which cancels the inter-layer interference (ILI) using the minimum mean square error (MMSE) estimates of the information symbols, and a diversity combining module which extracts the diversity components from the clipping noise. We show that the soft SIC module alone can effectively suppress the ILI, leading to bit error rates (BERs) resembling those of a genie receiver which is free of error propagation. It is also shown that the diversity combining receiver which combines the MMSE estimates with their diversity components outperforms the genie receiver by up to $2 \mathrm{~dB}$ at the BER of $10^{-6}$.
\end{abstract}

Index Terms-Layered ACO-OFDM, Inter-Layer Interference, Successive Interference Cancellation, Diversity Combining.

\section{INTRODUCTION}

D UE to its high power and spectral efficiency, layered asymmetrically clipped optical orthogonal frequency division multiplexing (ACO-OFDM) is increasingly being studied in the area of optical wireless and fibre communications [1][2]. Unlike conventional optical orthogonal frequency division multiplexing (OFDM) schemes, the structure of layered ACO-OFDM imposes inter-layer interference (ILI) which is caused by asymmetrical clippings in each layer. Hence, the receiver for the layered ACO-OFDM must be able to cancel the ILI successively.

A conventional layered ACO-OFDM receiver is designed based on the fact that the signal dependent ILI created in a layer only falls onto the subcarriers included in the subsequent layers and the direct current (DC) component [3]. Thus, the approach taken by it is to demodulate the symbols sequentially from low layer to high layer, where, in each layer, hard decoding is applied in the demodulation followed by ILI reconstruction (using the hard decisions) and cancellation [3].

In the context of additive white Gaussian noise (AWGN) channel, the performance of a conventional receiver is limited by (1) error propagation and (2) additive noise. The error propagation occurs when symbols in a layer are incorrectly decoded. These symbols lead to erroneous reconstructed ILI which creates an additional interference to, other than removes the existing one from, the layers to be demodulated. Thus,

This work was supported under an Australian Research Council's (ARC) Discovery funding scheme (DP 160101693).

The authors are with the Global Big Data Technologies Centre, University of Technology Sydney, Sydney, Australia e-mail: \{Qian.Wang, Hang.Li, Xiaojing.Huang\}@uts.edu.au when a conventional receiver is used, the bit error rate (BER) degrades dramatically with increasing number of layers.

In order to prevent error propagation, soft successive interference cancellation (SIC) has been implemented in modern radio frequency wireless communication systems [4][5]. Unlike the hard decision based SIC, the soft SIC reconstructs the interference using the minimum mean square error (MMSE) estimates of the symbols. Thus, when conducted iteratively, it can gradually cancel the interference from the signals to be demodulated, leading to superior BER performance to its hard decision based counterpart.

Optical wireless systems are typically operated in the presence of intensive ambient light which induces additive white Gaussian distributed shot noise [6]. The conventional (single layer) ACO-OFDM receiver demodulates symbols from the odd frequencies only and thus suffers a 3-dB penalty against a bi-polar OFDM signal [7]. It was later shown that the BER of ACO-OFDM could theoretically be improved by up to $3 \mathrm{~dB}$ using either diversity combining [8] or noise clipping [9]. These approaches, however, cannot be applied directly to layered ACO-OFDM because the correct recovery of the signals in each layer is prevented by the ILI. In [10], an iterative receiver is proposed to improve the BER of layered ACO-OFDM, where hard decisions are made assisted by noise clipping to cancel the ILI. While improved BERs are achieved, the error propagation is yet to be addressed.

In this letter, we present a diversity combining receiver employing soft SIC to combat the error propagation and additive noise for layered ACO-OFDM. We show that the soft SIC alone can effectively suppress the error propagation, leading to BERs resembling those of a genie receiver which is free of error propagation. It is also shown that the proposed receiver which combines the outputs of the SIC and their diversity components achieves an improved BER, outperforming the genie receiver by up to $2 \mathrm{~dB}$ at the BER of $10^{-6}$.

\section{LAYERED ACO-OFDM}

An $L$-layer ACO-OFDM modulator, $L \leq \log _{2} N$, which converts complex bipolar symbols, $\boldsymbol{X}=$ $\left[0, X_{1}, \cdots, X_{N / 2-1}, 0, X_{N / 2-1}^{*}, \cdots, X_{1}^{*}\right]^{T}$ to real nonnegative intensity signals, $\boldsymbol{s}=\left[s_{0}, s_{1}, s_{2}, \cdots, s_{N-1}\right]^{T}$, consists of $L$ single layer ACO-OFDM modulators (layers), where independent $M$-QAM symbols, $X_{1}, \cdots, X_{N / 2-1}$, with $E\left(\left|X_{n}\right|^{2}\right)=\epsilon$, are loaded with Hermitian symmetry. Here, $N$ denotes the size of the IFFT at each modulator and the superscripts, $[\cdot]^{T}$ and $[\cdot]^{*}$, represent the vector/matrix 
transpose and complex conjugate, respectively. Unlike conventional ACO-OFDM [7], the inputs to the $l$ th, $l \leq L$, modulator (layer), denoted by $\boldsymbol{X}_{l}=\left[X_{l, 0}, X_{l, 1}, \cdots, X_{l, N-1}\right]^{T}$, is related to the input symbol, $\boldsymbol{X}$, by

$$
X_{l, n}=\left\{\begin{array}{lc}
X_{n}, & \text { if } n \in G_{l} \\
0, & \text { otherwise }
\end{array}\right.
$$

where $G_{l}$ denotes the $l$ th subcarrier group (SCG) which includes those subcarriers with indexes dividable by $2^{l-1}$ but not dividable by $2^{l}$ [3]. Eq. (1) confirms that the conventional ACO-OFDM corresponds to the first layer modulator which loads symbols only onto the odd subcarriers.

The IFFT in the $l$ th layer generates a sequence, $\boldsymbol{x}_{l}=$ $\left[x_{l, 0}, x_{l, 1}, \cdots, x_{l, N-1}\right]^{T}$, in time domain, given by

$$
x_{l, k}=\frac{1}{\sqrt{N}} \sum_{n=0}^{N-1} X_{l, n} e^{j \frac{2 \pi}{N} k n}, \text { for } 0 \leq k \leq N-1,
$$

which produces the output of the modulator, $\boldsymbol{s}_{l}=$ $\left[s_{l, 0}, s_{l, 1}, \cdots, s_{l, N-1}\right]^{T}$, through clipping the negative value at zero to give

$$
s_{l, k}=\frac{1}{2} x_{l, k}+\frac{1}{2}\left|x_{l, k}\right|=\left\{\begin{array}{ll}
x_{l, k}, & \text { if } x_{l, k} \geq 0 \\
0, & \text { otherwise }
\end{array} .\right.
$$

Denote the FFT of $\boldsymbol{s}_{l}$ by $\boldsymbol{S}_{l}$. Then (3) indicates that $\boldsymbol{S}_{l}$ consists of two components: the information symbols with half amplitude loaded onto the subcarriers included in the $l$ th SCG and the clipping noise which is given by half of the absolute value of $\boldsymbol{x}_{l}$. As the time sequence, $\boldsymbol{x}_{l}$, consists of $2^{l-1}$ identical sub-sequences, each being anti-periodic [3], the clipping noise, $\left|\boldsymbol{x}_{l}\right| / 2$, is $2^{l}$-folded periodic in time domain. This indicates that after being converted using FFT, the clipping noise only falls onto the subcarriers allocated to higher layers and the DC.

The output of the $L$-layer ACO-OFDM modulator, $s$, is the sum of those of all the single layer modulators, i.e.

$$
s_{k}=\sum_{l=1}^{L} s_{l, k}, \text { for } 0 \leq k \leq N-1,
$$

with the electrical power, $P_{\text {elec}}$, given by ${ }^{1}[11]$

$$
P_{\mathrm{elec}}=E\left(s_{k}^{2}\right)=\frac{1}{2} \sum_{l=1}^{L} \sigma_{l}^{2}+\frac{1}{\pi} \sum_{l_{1} \neq l_{2}}^{L} \sigma_{l_{1}} \sigma_{l_{2}},
$$

where $\sigma_{l}^{2}$ denotes the electrical power of $x_{l, k}$, given by $E\left(x_{l, k}^{2}\right)$. Denote the bit rate of a single layer ACO-OFDM by $r_{1}$ bits per second. Then the rate of an $L$-layer signal employing identical constellation can be expressed as [1]

$$
r_{L}=\left(1+\frac{1}{2}+\cdots+\frac{1}{2^{L-1}}\right) r_{1}=2\left(1-\left(\frac{1}{2}\right)^{L}\right) r_{1} .
$$

Therefore, the average electrical energy per bit, $E_{\mathrm{b}, \text { elec }}$, is given by $E_{\mathrm{b}, \text { elec }}=P_{\text {elec }} / r_{L}[7]$.

\footnotetext{
${ }^{1}$ Eq. (5) holds strictly if no less than 16 subcarriers are loaded with information symbols in the Lth SCG [11]. Otherwise, it is a close approximation.
}

\section{Diversity Combining RECEIVER WITH SOFT SIC}

In this section, we describe the diversity combining receiver that provides improved BER performance. Recent research has shown that although DC offset can cause a diversity combining receiver to fail, its performance can be regained using DC estimation [12]. Therefore, in this paper, we ignore the effect of DC offset and assume identical zero levels at the transmitter and receiver. The received signal can thus be expressed, in a compact form, as

$$
y=s+w
$$

where $\boldsymbol{w}$ denotes the vector of additive white Gaussian noise with single-sided power spectral density, $N_{0}$. Assuming an ideal anti-aliasing filter is used, which has a bandwidth of $B$ equalling that of the wanted signal, the noise power, $\sigma_{\mathrm{w}}^{2}$, is then given by $\sigma_{\mathrm{w}}^{2}=N_{0} B$.

\section{A. Soft Successive Interference Cancellation}

In this section, we recover the symbols in each layer using soft SIC. Unlike the hard-decoding based SIC, the soft SIC employs the MMSE estimates of the symbols to reconstruct the ILI. Thus, as no hard decisions are made in the process of interference cancellation, error propagation is avoided.

The way the SCGs are created leads to a unique interference pattern in layered ACO-OFDM. The clipping noise falls only onto the higher layers and the DC component. This is reflected by the FFT of the received signal, $\boldsymbol{y}$ given by (7), as

$$
\boldsymbol{Y}=\frac{1}{2} \boldsymbol{X}_{1}+\frac{1}{2} \boldsymbol{C}_{1}+\cdots+\frac{1}{2} \boldsymbol{X}_{l}+\frac{1}{2} \boldsymbol{C}_{l}+\cdots+\frac{1}{2} \boldsymbol{X}_{L}+\frac{1}{2} \boldsymbol{C}_{L}+\boldsymbol{W},
$$

where $\boldsymbol{C}_{l}$ and $\boldsymbol{W}$ denote the clipping noise created in the $l$ th layer and the FFT of $\boldsymbol{w}$, respectively. Using (3), we can see that $\boldsymbol{C}_{l}$ is the FFT of $\left|\boldsymbol{x}_{l}\right|$, which equals zero on the subcarriers included in the first $l$ SCGs. Therefore, the recovery must start from the first layer in which the information carrying subcarriers (included in the 1st SCG) are free of interference. Denote the MMSE esitmate of $\boldsymbol{X}_{1}$ by $\hat{\boldsymbol{X}}_{1}$. The estimate of the clipping noise, $\hat{\boldsymbol{C}}_{1}$, can be reconstructed through converting $\hat{\boldsymbol{X}}_{1}$ to time domain to give $\hat{\boldsymbol{x}}_{1}$ followed by taking its absolute values (see (3)) and an FFT. Then the estimates, $\hat{\boldsymbol{X}}_{1}$ and $\hat{\boldsymbol{C}}_{1}$, are subtracted from the received signal to yield $\hat{\boldsymbol{Y}}_{1}$ which is used to estimate $\hat{\boldsymbol{X}}_{2}$ and $\hat{\boldsymbol{C}}_{2}$ in layer 2 . The whole procedure ends when the symbols in all the layers are estimated.

For the recovery in the $l$ th layer, the observation for estimation can be expressed as

$$
\hat{\boldsymbol{Y}}_{l-1}=\frac{1}{2} \boldsymbol{X}_{l}+\frac{1}{2} \boldsymbol{C}_{l}+\cdots+\frac{1}{2} \boldsymbol{X}_{L}+\frac{1}{2} \boldsymbol{C}_{L}+\boldsymbol{W}+\boldsymbol{Z}_{l-1},
$$

where $Z_{l-1}$ denotes the accumulated estimation error resulted from the previous layers, given by

$$
\boldsymbol{Z}_{l-1}=\frac{1}{2} \sum_{m=1}^{l-1}\left(\boldsymbol{X}_{m}-\hat{\boldsymbol{X}}_{m}\right)+\frac{1}{2} \sum_{m=1}^{l-1}\left(\boldsymbol{C}_{m}-\hat{\boldsymbol{C}}_{m}\right) .
$$

In order to derive the MMSE estimate of $\boldsymbol{X}_{l}$, the probability density function (PDF) of the estimation error, $\sum_{m=1}^{l-1}\left(\boldsymbol{C}_{m}-\hat{\boldsymbol{C}}_{m}\right) / 2$, on the subcarriers included in the $l$ th SCG is needed. However, as will be shown in the simulation results, 
the exact PDF can only provide negligible improvement on the BER. Therefore, in this paper, we ignore the estimation error, $Z_{l-1}$. Then the observation on the $n$th subcarrier, $n \in G_{l}$, can be expressed as

$$
\hat{Y}_{l-1, n}=\frac{1}{2} X_{n}+W_{n},
$$

where $W_{n}$ denotes the $n$th element of $\boldsymbol{W}$, having a Gaussian distribution with zero mean and variance $\sigma_{\mathrm{w}}^{2}$.

The MMSE estimate of $X_{n}$ with an observation, $\hat{Y}_{l-1, n}$, is given by the expectation of $X_{n}$ conditioned on $\hat{Y}_{l-1, n}$, i.e. $\hat{X}_{n}=E\left(X_{n} \mid \hat{Y}_{l-1, n}\right)$ [13]. Therefore, for an $M$-QAM information symbol, $X_{n}=a_{n}+j b_{n}$, the estimates of its real and imaginary parts, $\hat{a}_{n}$ and $\hat{b}_{n}$, can be expressed as [4]

$$
\hat{a}_{n}=\frac{\sum_{a \in \mathcal{M}_{\mathrm{R}}} a \exp \left[-\left(\mathfrak{R}\left(\hat{Y}_{l-1, n}\right)-a\right)^{2} /\left(4 \sigma_{\mathrm{w}}^{2}\right)\right]}{\sum_{a \in \mathcal{M}_{\mathrm{R}}} \exp \left[-\left(\mathfrak{R}\left(\hat{Y}_{l-1, n}\right)-a\right)^{2} /\left(4 \sigma_{\mathrm{w}}^{2}\right)\right]}
$$

and

$$
\hat{b}_{n}=\frac{\sum_{b \in \mathcal{M}_{\mathrm{I}}} b \exp \left[-\left(\mathfrak{J}\left(\hat{Y}_{l-1, n}\right)-b\right)^{2} /\left(4 \sigma_{\mathrm{w}}^{2}\right)\right]}{\sum_{b \in \mathcal{M}_{\mathrm{I}}} \exp \left[-\left(\mathfrak{J}\left(\hat{Y}_{l-1, n}\right)-b\right)^{2} /\left(4 \sigma_{\mathrm{w}}^{2}\right)\right]},
$$

respectively, leading to $\hat{X}_{n}=\hat{a}_{n}+j \hat{b}_{n}$, where $\mathfrak{R}(\cdot)$ and $\mathfrak{J}(\cdot)$ denote the real and imaginary parts of a complex number, and $\mathcal{M}_{\mathrm{R}}$ and $\mathcal{M}_{\mathrm{I}}$, the sets that include all the possible values for the real and imaginary parts of the constellation.

For the subcarriers that are not included in the $l$ th SCG, the corresponding elements in $\hat{\boldsymbol{X}}_{l}$ are forced to zero. Once the symbols in all the layers have been estimated, the estimates are sent to maximum likelihood (ML) decoders which make hard decisions of the symbols transmitted.

Comparing the soft SIC with the conventional receiver, we can see that the two methods share equal number of (I)FFTs, subtraction devices and ML decoders. The only overhead of soft SIC is the MMSE estimators which evaluate the estimates given by (12) and (13). As significant improvement shown in the simulation results can be achieved, the slight increase of complexity is reasonably acceptable.

\section{B. Diversity Combining Receiver}

The receiver described above estimates the information symbols in the $l$ th layer using only the subcarriers included in the $l$ th SCG. The clipping noise on the subsequent layers, however, are not used, although it contains useful information for demodulation (see (3)). In this section, we recover the clipping noise created in each layer to enable a high performance diversity combining receiver. In order to distinguish from that reconstructed, we denote the estimate of the clipping noise obtained from the subsequent layers by $\overline{\boldsymbol{C}}_{l}, l=1, \cdots, L$.

Given the estimates, $\hat{\boldsymbol{X}}_{l}, l=1, \cdots, L$ and $\hat{\boldsymbol{C}}_{l}, l=1, \cdots, l-$ $1, l+1, \cdots L$, returned from the SIC module, using (8), an estimate of $\boldsymbol{C}_{l}$, given by $\overline{\boldsymbol{C}}_{l}$, can be derived as

$$
\overline{\boldsymbol{C}}_{l}=2 \boldsymbol{Y}-\sum_{m=1}^{L} \hat{\boldsymbol{X}}_{m}-\sum_{m \neq l}^{L} \hat{\boldsymbol{C}}_{m},
$$

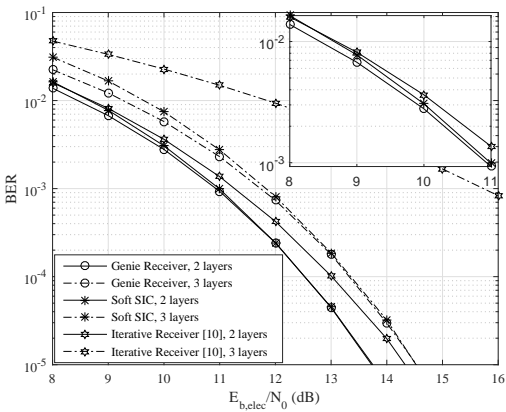

Fig. 1. BER achieved by soft SIC.

where the elements of $\overline{\boldsymbol{C}}_{l}$ corresponding to the subcarriers included in the first $l$ SCGs are forced to zero. Note that although representing an alternative of $\hat{\boldsymbol{C}}_{l}, \overline{\boldsymbol{C}}_{l}$ include the additive noise on the subcarriers included in the $(l+1)$ th, $\cdots, L$ th SCGs, whereas $\hat{\boldsymbol{C}}_{l}$ depends only on the noise on those in the $l$ th SCG and the estimation errors accumulated in the previous layers. Therefore, diversity receiver that combines the information carried by $\hat{\boldsymbol{X}}_{l}$ with its clipping noise, $\overline{\boldsymbol{C}}_{l}$, can be applied to suppress the additive noise [8].

Denote the IFFT of $\hat{\boldsymbol{X}}_{l}$ and $\overline{\boldsymbol{C}}_{l}$ by $\hat{\boldsymbol{x}}_{l}$ and $\overline{\boldsymbol{x}}_{l}$, respectively. As the relationship $\overline{\boldsymbol{x}}_{l}=\left|\hat{\boldsymbol{x}}_{l}\right|$ holds in the absence of noise, a diversity component, $\boldsymbol{x}_{l}^{\mathrm{d}}$, can be formed by element-wisely flipping $\overline{\boldsymbol{x}}_{l}$ in accordance with the signs of $\hat{\boldsymbol{x}}_{l}$ [8][12], i.e.

$$
\boldsymbol{x}_{l}^{\mathrm{d}}=\overline{\boldsymbol{x}}_{l} \otimes \operatorname{sgn}\left(\hat{\boldsymbol{x}}_{l}\right),
$$

where $\operatorname{sgn}(\cdot)$ denotes the sign function returning 1 with a nonnegative input and -1 otherwise and " $\otimes$ ", the element-wise multiplication. The two signals, $\hat{\boldsymbol{x}}_{l}$ and $\boldsymbol{x}_{l}^{\mathrm{d}}$, are then combined using a set of combining coefficients, $\alpha_{l}, l=1, \cdots, L$, to generate a signal, $\boldsymbol{x}^{\left(\alpha_{l}\right)}[8][12]$,

$$
\boldsymbol{x}_{l}^{\left(\alpha_{l}\right)}=\left(1-\alpha_{l}\right) \hat{\boldsymbol{x}}_{l}+\alpha_{l} \boldsymbol{x}_{l}^{\mathrm{d}}, \quad 0 \leq \alpha_{l} \leq 1,
$$

which leads to improved BER performance.

\section{Simulation Results}

In this section, we present the simulated BER results and compare them with those achieved by the existing receiver [10]. The layered ACO-OFDM signal with a bandwidth of 2 $\mathrm{MHz}$ is configured to modulate 4-QAM symbols onto $N=256$ subcarriers and transmitted with unit electrical power, i.e. $P_{\text {elec }}=1$. Assuming perfect synchronization, the receiver samples the received signal with Nyquist sampling rate. The noise power is set as a variable to generate the values for $E_{\mathrm{b}, \text { elec }} / N_{0}$ ranging from $8-16 \mathrm{~dB}$. In order to give insights on the error propagation, a genie receiver is employed, which demodulates the received signal using hard decoding and can correctly reconstruct and completely cancel the ILI regardless of decoding errors. Thus, it is free of error propagation.

\section{A. BER Performance of Soft SIC}

Fig. 1 shows the simulated BERs achieved by the estimates, $\hat{\boldsymbol{X}}_{l}, l=1, \cdots, L$, returned by the soft SIC, the genie receiver and the hard-decoding assisted iterative receiver [10]. From 


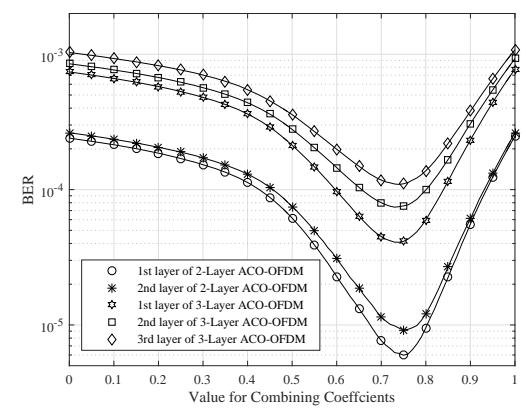

Fig. 2. BER as a function of the combining coefficients.

this figure, it can be seen that the soft SIC can achieve a BER close to that of a genie receiver for both double- and triple-layer ACO-OFDM. This means that the soft SIC can effectively suppress the error propagation. The small gaps between the soft SIC and genie receiver indicate that an exact PDF of the estimation error will not significantly improve the performance. The BER of a hard-decoding assisted iterative receiver [10] with three iterations is also plotted for comparison. As shown in the figure, because of the use of hard decoding, error propagation constantly occurs, leading to the resulting BER deviating from that of the genie receiver.

\section{B. BER Performance of Diversity Combining Receiver}

As shown in (16), the coefficients, $\alpha_{l}, l=1, \cdots, L$, indicate different weightings of $\hat{\boldsymbol{x}}_{l}$ and $\boldsymbol{x}_{l}^{\mathrm{d}}$ in the combined signal. A small value for $\alpha_{l}$ means the SIC output dominates whereas a large value, the diversity component. The optimum coefficients depend on the noise distribution and power in each signal. The SIC output, $\hat{\boldsymbol{X}}_{l}$, is impaired only by the estimation error which is not Gaussian distributed. Thus, it alone cannot achieve the best performance as the ML decoder typically used in wireless communications is optimum only in the presence of Gaussian noise. The clipping noise, $\overline{\boldsymbol{C}}_{l}$, on the contrary, includes Gaussian noise which remains to be Gaussian in the flipping, (15), and thus matches the ML decoder. However, the signal-to-noise ratio of diversity component is relatively low compared with the SIC output, because of the larger accumulated estimation error. Hence, the optimum value for the coefficients must balance the noise distribution and power.

Fig. 2 shows the BER of various layers as a function of the combining coefficients with $E_{\mathrm{b} \text {,elec }} / N_{0}=12 \mathrm{~dB}$. As shown in the figure, neither the SIC outputs nor the diversity components alone can achieve the minimum BER. An exhaustive search is conducted to find the optimum coefficients which are shown for double-layer ACO-OFDM, $\alpha_{1}^{\text {opt }}=0.75$ and $\alpha_{2}^{\text {opt }}=0.75$, and for triple-layer, $\alpha_{1}^{\text {opt }}=0.74, \alpha_{2}^{\text {opt }}=0.75$ and $\alpha_{3}^{\text {opt }}=0.74$. The BERs of diversity combining receiver using the optimum coefficients are shown in Fig. 3, where the BERs of genie receiver are plotted for comparison. As shown in the figure, we can see a significant improvement achieved by the diversity combining receiver which outperforms the genie receiver by approximately $2 \mathrm{~dB}$ at the BER of $10^{-6}$.

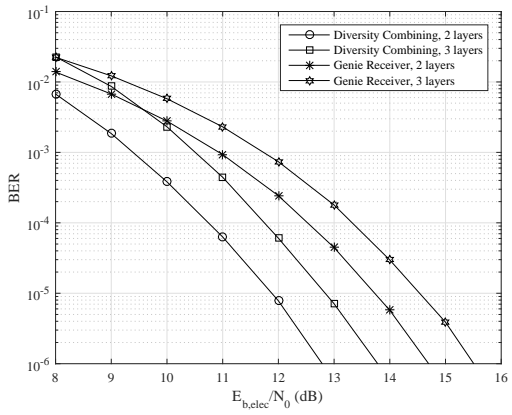

Fig. 3. BER of diversity combining receiver.

\section{CONCLUSION}

We have studied a high performance diversity combining receiver for layered ACO-OFDM. The soft SIC is applied to recover the information symbols and ILI, and is shown to be significantly effective to suppress the error propagation. The simulated BER shows that the outputs of soft SIC alone can achieve a similar performance with that of an interference-free genie receiver. It is also shown that a BER improvement up to $2 \mathrm{~dB}$ over the genie receiver can be achieved when these outputs are combined with their diversity components.

\section{REFERENCES}

[1] Q. Wang, C. Qian, X. Guo, Z. Wang, D. G. Cunningham, and I. H. White, "Layered ACO-OFDM for intensity-modulated direct-detection optical wireless transmission," Optics Express, vol. 23, pp. 12382-12393, May 2015.

[2] A. J. Lowery, "Comparisons of spectrally-enhanced asymmetricallyclipped optical OFDM systems," Optics Express, vol. 24, pp. 3950-3966, Feb. 2016.

[3] L. Chen, B. Krongold, and J. Evans, "Successive Decoding of AntiPeriodic OFDM Signals in IM/DD Optical Channel," in Int. Conf. on Communications, Cape Town, 2010, pp. 1-6.

[4] A. Lampe and J. B. Huber, "On Improved Multiuser Detection with Iterated Soft Decision Interference Cancellation," in ICC 1999, Comm. Theory Mini-Conference, Vancouver, 1999, pp. 172-176.

[5] M. Wu, B. Yin, G. Wang, C. Dick, J. R. Cavallaro and C. Studer, "Large-Scale MIMO Detection for 3GPP LTE: Algorithms and FPGA Implementations," IEEE J. Sel. Topics Signal Process., vol. 8, pp. 916929, Oct. 2014.

[6] J. M. Kahn and J. R. Barry, "Wireless infrared communications," Proc. IEEE, vol. 85, pp. 265-298, Feb. 1997.

[7] J. Armstrong and B. J. C. Schmidt, "Comparison of asymmetrically clipped optical OFDM and DC-biased optical OFDM in AWGN," IEEE Commun. Lett., vol. 12, pp. 343-345, May 2008.

[8] L. Chen, B. Krongold, and J. Evans, "Diversity Combining for Asymmetrically Clipped Optical OFDM in IM/DD Channels," in IEEE Globecom, Honolulu, HI, 2009, pp. 1-6.

[9] K. Asadzadeh, A. Dabbo, and S. Hranilovic, "Receiver design for asymmetrically clipped optical OFDM," in Globecom Workshops 2011 (GW'11), Houston, TX, 2011, pp. 777-781.

[10] Q. Wang, Z. Wang, X. Guo, and L. Dai, "Improved Receiver Design for Layered ACO-OFDM in Optical Wireless Communications," IEEE Photon. Technol. Lett., vol. 28, pp. 319-322, Feb. 2016.

[11] L. Chen, B. Krongold, and J. Evans, "Performance Analysis for Optical OFDM Transmission in Short-Range IM/DD Systems," IEEE/OSA J. Lightw. Technol., vol. 30, pp. 974-983, Apr. 2012.

[12] M. Tahar, T. Q. Wang, M. F. G. Medina, O. Gonzalez, and J. Armstrong, "Experimental Demonstration of Diversity Combining for Asymmetrically Clipped Optical OFDM," IEEE Commun. Lett., vol. 20, pp. 906-909, May 2016.

[13] A. H. Sayed, Adaptive Filters. Hoboken, New Jersey: John Wiley \& Sons, Inc., 2008. 